\title{
LASERAKUPUNKTURFORSCHUNG: CHINA VOR ÖSTERREICH UND USA
}

\section{Liebe Kolleginnen und Kollegen,}

Um es gleich vorweg zu nehmen: Zur Laserakupunktur gibt es immer mehr SCI/PubMed gelistete Forschungsarbeiten. Insgesamt finden sich dazu in der wohl wichtigsten medizinischen Datenbank (www.pubmed.gov) mit Stand August 2018 knapp über 900 Arbeiten. Wenngleich die Praxis der Laserakupunktur in China noch immer in den Kinderschuhen zu stecken scheint, so nimmt im internationalen wissenschaftlichen Ranking bezüglich veröffentlichter Forschungsarbeiten dennoch auch hier China die erste Stelle ein. Insgesamt wurden 225 wissenschaftliche Arbeiten zu Thematik Laserakupunktur unter Autorenbeteiligung aus China verfasst. Sieht man sich Details dazu an, so ist doch erwähnenswert, dass 44 Arbeiten davon unter Beteiligung des TCM-Forschungszentrums Graz (Leiter: G. Litscher) entstanden sind. Überhaupt nimmt Österreich in dieser Statistik in Ergänzung zu China eine federführende Rolle ein.

Bemerkenswert ist die Tatsache, dass die österreichischen ForscherInnen insgesamt mehr Arbeiten als die USA und Deutschland zusammen in diesem Forschungsbereich veröffentlicht haben und dass es in der Schweiz bislang nur sehr wenige referenzierte Arbeiten dazu gibt. Geht man bei dieser Betrachtung noch etwas in die Tiefe, so erkennt man, dass von den 98 publizierten Arbeiten aus Österreich ein sehr hoher Prozentsatz von Grazer ForscherInnen des TCM-Forschungszentrums stammt ( $\mathrm{n}=$ 91). Es ist also nicht übertrieben, wenn man feststellt, dass sich Graz zu einem Hotspot der Laserakupunkturfor- schung entwickelt hat und eben dort gemeinsam mit den VertreterInnen der ISLA transkontinental die Weichen für zukünftige Schwerpunkte gestellt werden.

Ein wichtiger Punkt wird beispielsweise die Entwicklung der automatisierten, individuellen Dosisanpassung bei der Laserakupunktur sein, die derzeit noch in keinem kommerziell erhältlichen Gerät implementiert ist. Immer wieder wird vom Leiter des TCM-Forschungszentrums Graz in Vorträgen darauf hingewiesen, dass die Ideenkonstrukte dafür vorhanden sind, aber bislang keine Firma diese zukunftsweisenden Aspekte in einzelne Gerätekomponenten implementieren möchte. Bleibt zu hoffen, dass diese Umsetzung von industriellen PartnerInnen erfolgt, die das Potenzial erkennen und sich nicht nur von eminenzbasierter, sondern eben auch evidenzbasierter Forschung überzeugen lassen.

Ende dieses Jahres gibt es wieder die ISLA-AsienKonferenz in Bangkok (siehe ISLA-Vorschau). Laserakupunktur ist im Begriff, auch in Thailand einen besonderen Aufschwung zu erfahren und daher wird dieser in der Konferenz auch ein besonderer Stellenwert gewidmet werden.

Wir würden uns freuen, Sie persönlich in Bangkok begrüßen zu dürfen. Es wartet ein spannender Event auf Sie.

Viel Freude beim Lesen dieser Ausgabe!

Mit freundlichen Grüßen

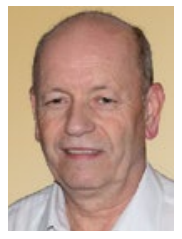

Dr. med. Dipl. Chem. Michael Weber

President for Medical and Clinical Applications der International Society for Medical Laser Applications (ISLAtranscontinental)

Sohnreystrasse 4

37697 Lauenförde

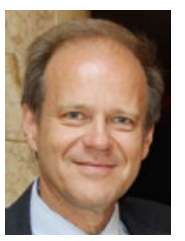

Univ.-Prof. Prof. h.c. DI Dr. techn.

Dr. scient. med. Gerhard Litscher

President for Science and Research der International Society

for Medical Laser Applications (ISLAtranscontinental)

Medizinische Universität Graz, Auenbruggerplatz 39,

EG19, A-8036 Graz, Tel: +43 316/385-83907

E-Mail:gerhard.litscher@medunigraz.at,Internet:http://litscher.info 